\title{
THE HERTZSPRUNG-RUSSELL DIAGRAMS FOR HOT STARS AND O- AND T- ASSOCIATIONS
}

\author{
I. M. KOPYLOV \\ Crimean Astrophysical Observatory, USSR
}

One of the most important points in the study of the problem of the origin and evolution of stars is a detailed analysis of the structure of the HR diagram as a whole, and the structure of its separate sequences.

The differences in the morphological and spatial-kinematical characteristics of the stars comprising the various sequences have an evolutionary character and are the result of differences in the origin and evolution of stars.

The position of a star in the HR diagram is determined primarily by its mass, chemical composition and age. In view of this the HR diagram provides a natural basis for the theoretical development of problems of the origin and evolution of stars. From this point of view a detailed study of the distribution of the stars of the general galactic field and of separate spatial groups in the HR diagram (on the Sp, M plane) is undoubtedly of great significance.

The assumption that hot massive stars are young and the theory of the group origin of stars in the Galaxy induce us to devote special attention to a thorough study of the HR diagram for hot stars of high luminosity.

A rather promising approach to this question, in our opinion, is to construct most accurate HR diagrams for individual groups of hot stars and to compare these diagrams with the combined HR diagram for the largest possible number of such groups, as well as with the HR diagram for hot stars in general, irrespective of the spatial group to which they belong.

The galactic stellar field evidently represents a rather heterogeneous mixture of stars of different origin and different ages, belonging, perhaps, to no-longer existent spatial groups, such as star clouds, associations, clusters, etc.

We can therefore formulate two rather general hypotheses, which are independent of any definite conceptions as to the paths and rates of stellar evolution :

a) The general HR diagram for stars of the galactic field - presenting a mixture of stars of different origin and different ages - should differ from the HR diagram for separate spatial groups of stars, which undoubtedly in each case have a common origin and perhaps are of the same age.

b) The combined HR diagram for the greatest possible number of different groups must closely approach in form the diagram for the stars of the general galactic field. There will not necessarily be complete correspondence, since the stars of the field, on the average, are evidently of different age than the stars in the different groups existing at present.

Let us examine these hypotheses from the observational point of view. As a result of our work in 1953-57 we were able to develop a quantitative system of two-dimensional spectral classification of hot stars of the spectral classes $\mathrm{O}_{5}-\mathrm{B} 7$. In this system we then determined with a high degree of accuracy the absolute magnitudes and spectral classes of 238 stars in the interval $\mathrm{O}_{5}-\mathrm{B} 7$ (1958). 
The probable error in the determination of the absolute magnitudes of $\mathrm{O}$ and $\mathrm{B}$ stars in our system is equal on the average to $\pm 0^{\mathrm{m}}$.Io. The probable error in the determination of the spectral class is equal on the average to \pm 0.15 of the spectral subclass. Thus, the errors in the determination of stellar parameters required for plotting an accurate HR diagram for hot stars are sufficiently small and of the order of Io $\%$ for $\mathrm{M}_{v}$, and 2 to $3 \%$ for Sp $\left(\log \mathrm{T}_{\text {eff }}\right)$.

Using the values of $M_{v}$ and Sp obtained for 238 stars we plotted the HR diagram in the spectral range $\mathrm{O}_{5}-\mathrm{B} 7$. For the spectral range $\mathrm{B} 9-\mathrm{Fo}$ we plotted the diagram for stars fainter than $-I^{m}$ with known trigonometric parallaxes in the vicinity of the Sun. For stars of high luminosity in the spectral range $\mathrm{B} 8$ - Fo the value of $\mathrm{M}_{\mathrm{v}}$ was determined by indirect methods. The error in the determination of $\mathrm{M}_{\mathrm{v}}$ in the range $\mathrm{Bg}-\mathrm{Fo}$ is of the order of $\pm \mathrm{O}^{\mathrm{m}} .2$ $-\mathrm{o}^{\mathrm{m}} \cdot 3$; the error in the determination of $\mathrm{Sp}$ is of the order of $\pm 0.3-0.5$ of a spectral subclass.

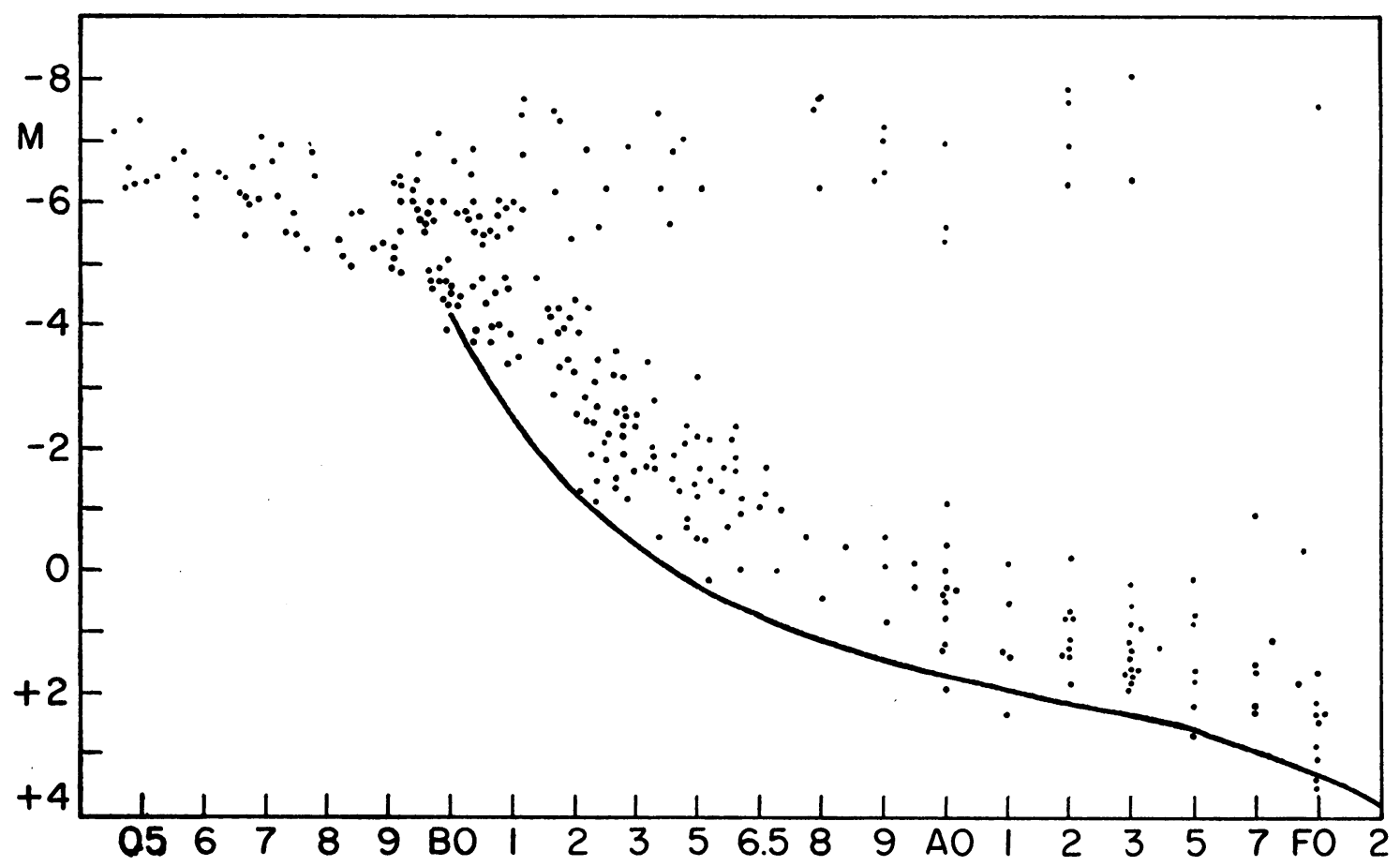

FIGURE I. The HR diagram for early-type stars, from spectroscopic and trigonometric parallaxes.

It should be observed that in the diagram shown (Fig. I) the distribution of stars in absolute magnitude is to a considerable degree distorted by the conditions of visibility in the Galaxy; with an increase in the luminosity the number of stars in the diagram is greater than the true number, since the diagram is plotted in general for stars brighter than a given limiting visible magnitude, which is different for different spectral ranges. For this reason Fig. I does not give a sufficiently exact idea of the relative numbers of stars of different luminosities, i.e. the luminosity function. A considerable number of stars in Fig. I, especially those of low luminosity, belong to the general galactic field.

We enumerate the most characteristic properties of the general HR diagram for stars of the range $\mathrm{O}_{5}-\mathrm{Fo}$. 
I. The existence of two partially overlapping bands of stars : - the band composed of stars of the luminosity classes III - V and extending almost diagonally from the upper left to the lower right of the HR diagram is, evidently, the main sequence of stars, taken in the broadest sense of the term. The width of this band varies from $2^{m}-3^{m}$ in the range $B_{2}$ Fo to $\leqslant 2^{\mathrm{m}}$ in the range $\mathrm{O}_{9}-\mathrm{BI}$. There is no sharp boundary between the stars of luminosity classes III, IV and V. The stars of luminosity class III are situated primarily near the upper boundary of the band, the stars of luminosity class V-near the lower boundary, while the middle of the band is occupied by a mixture of stars of luminosity classes III, IV and V. The second band consists of supergiants of the types Ia - II, of absolute magnitudes from $-5^{\mathrm{m}} .5$ to $-7^{\mathrm{m}} \cdot 5$; the width of the band is about $2^{\mathrm{m}}$. The two bands partially overlap and then merge in the region of spectral classes $\mathrm{O} 9-\mathrm{BI}$.

2. The impression is created that the stars of the classes $\mathrm{O}_{5}-\mathrm{O} 8$ are a continuation of the main sequence-the band consisting of stars of the luminosity classes III - V.

3. Stars of the classes 07.8 - O9.I, brighter than $-5^{\mathrm{m} .8}$, are absent. The band of supergiants of type Ia - II commences only at spectral subclass $\mathrm{O}_{9}$ and extends in the direction of the later spectral classes. The absence, or at any rate, very considerable shortage of stars

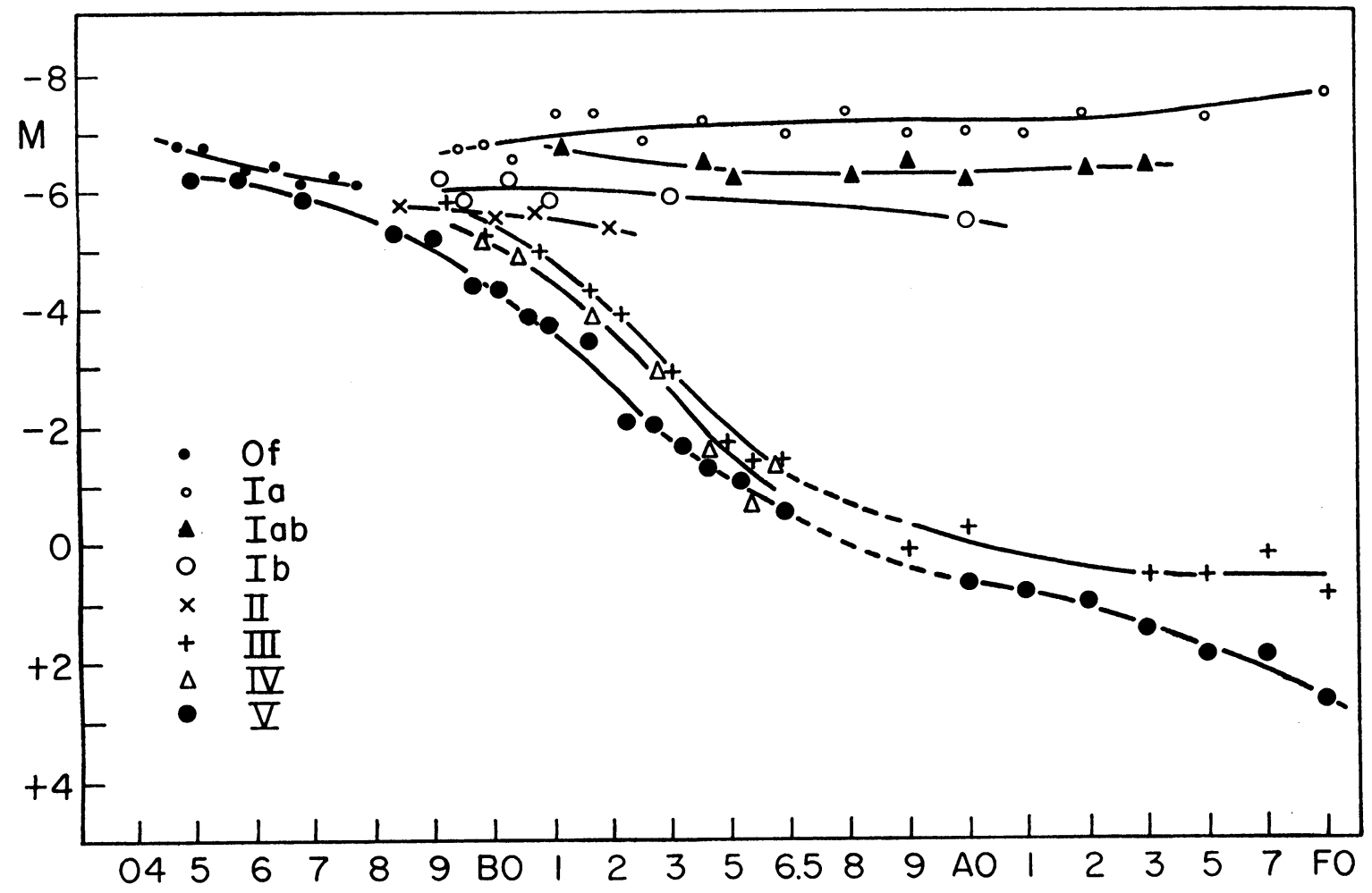

FIGURE 2. Absolute-magnitude calibration of the luminosity classes of early-type stars classified on the MK system.

brighter than $-6^{m}$ in the interval $08-O 9$ is confirmed by the analysis of the data in the catalogue of blue giants by Morgan, Whitford and Code (I955).

4. Stars of $\mathrm{B}_{2}$ and later classes of absolute magnitudes from $-4^{\mathrm{m}} \cdot 5\left(-3^{\mathrm{m}} \cdot 5\right)$ to $-5^{\mathrm{m}} \cdot 5$ are absent; there are also no stars of magnitude $M$ from $-I^{m}$ to $-5^{\mathrm{m}} \cdot 5$ in the range $B 8$ to Fo. 
5. The sequence of variable stars of the $\beta$ Canis Majoris type (II stars) lies near the upper boundary of the band III - V running approximately parallel to it.

The data used in plotting Fig. I can also be utilized to carry out a new, rather detailed calibration of the luminosity classes of the MK system for stars in the range O5 - Fo (Fig. 2).

It is interesting to consider the possibility of representing the general HR diagram obtain-

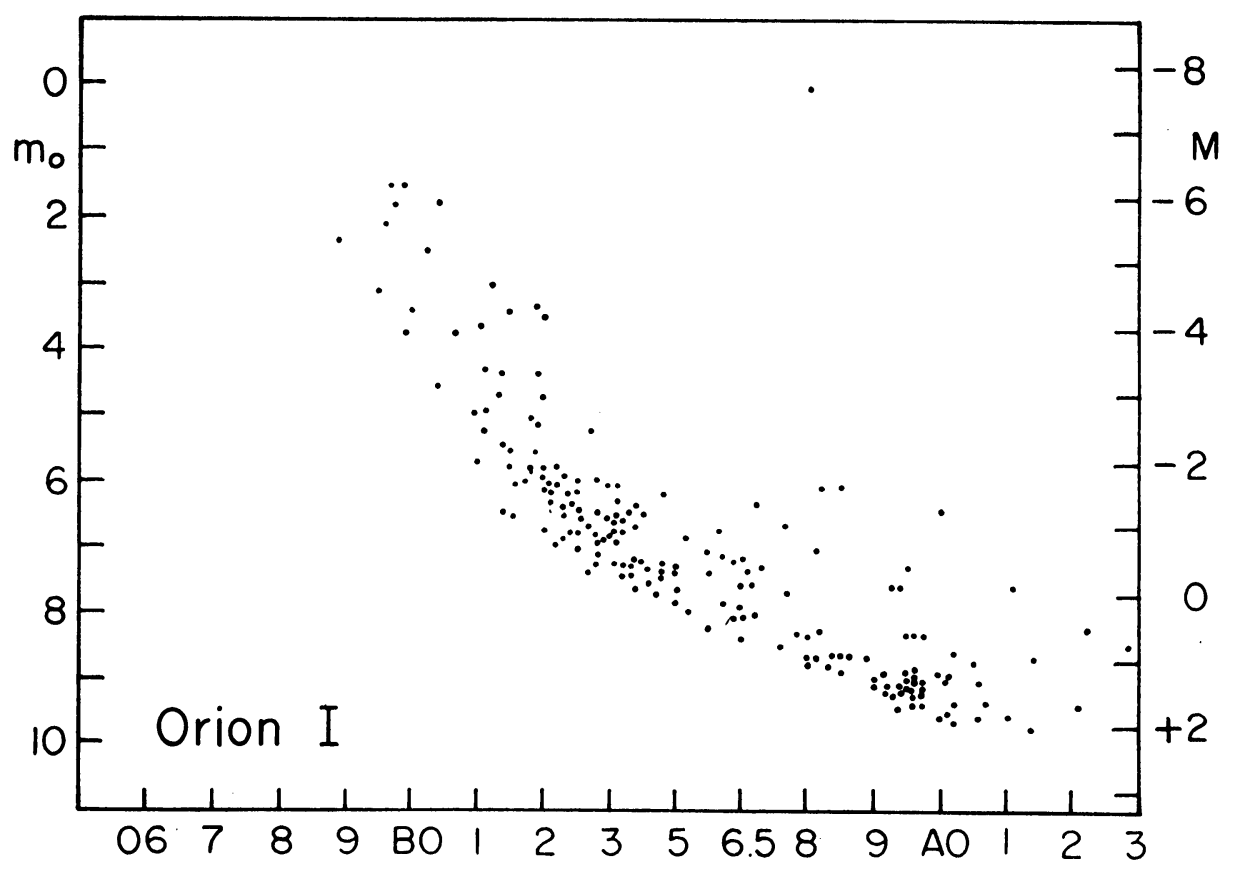

FIgURE 3. The HR diagram for the association, Orion I.

ed for hot stars by a series of corresponding diagrams for a number of definite spatial groups containing stars in the range $\mathrm{O}_{5}-\mathrm{Fo}$.

Utilizing the published data, we plotted HR diagrams for I3 spatial groups (clusters and associations). For most of these we determined the distance moduli from the stars composing them and having quantitatively determined values of $\mathrm{M}_{\mathrm{v}}$ on our system.

Figures 3-5 represent HR diagrams for certain stellar groups : Orion I, Perseus I, $\alpha$ Persei, NGC 2362.

The association Perseus $I$ is characterized by a strong band of $B$ and A supergiants, and over a dozen supergiants of class M. Supergiants of the classes F-K are absent. The presence of individual cool supergiants in the associations is a rather common phenomenon.

Stars of early class $\mathrm{O}$ are rather rare in associations. As a rule, associations contain one or two stars of class $O$. However, there are associations which contain about Io early O-stars each; in particular, the associations Mon I, B, Cas V (near IC I805), Cas VI (near IC I848.

The combined HR diagram for I3 spatial groups is given in Fig. 6. The comparison of Figures $I$ and 6 leaves no doubt that they are similar in their general features. Fig. 6 also fully confirms our conclusions regarding the properties of the general HR diagram for hot stars given in Fig. I. It appears, in addition, that the stars of the "main sequence" (the band 
of stars of luminosity classes III - V) displays a considerable concentration towards the lower boundary. The densities of stars in the Sp, Mv plane near the upper and lower boundaries of the band differ by a factor of at least ten. The lower boundary of the main sequence, in

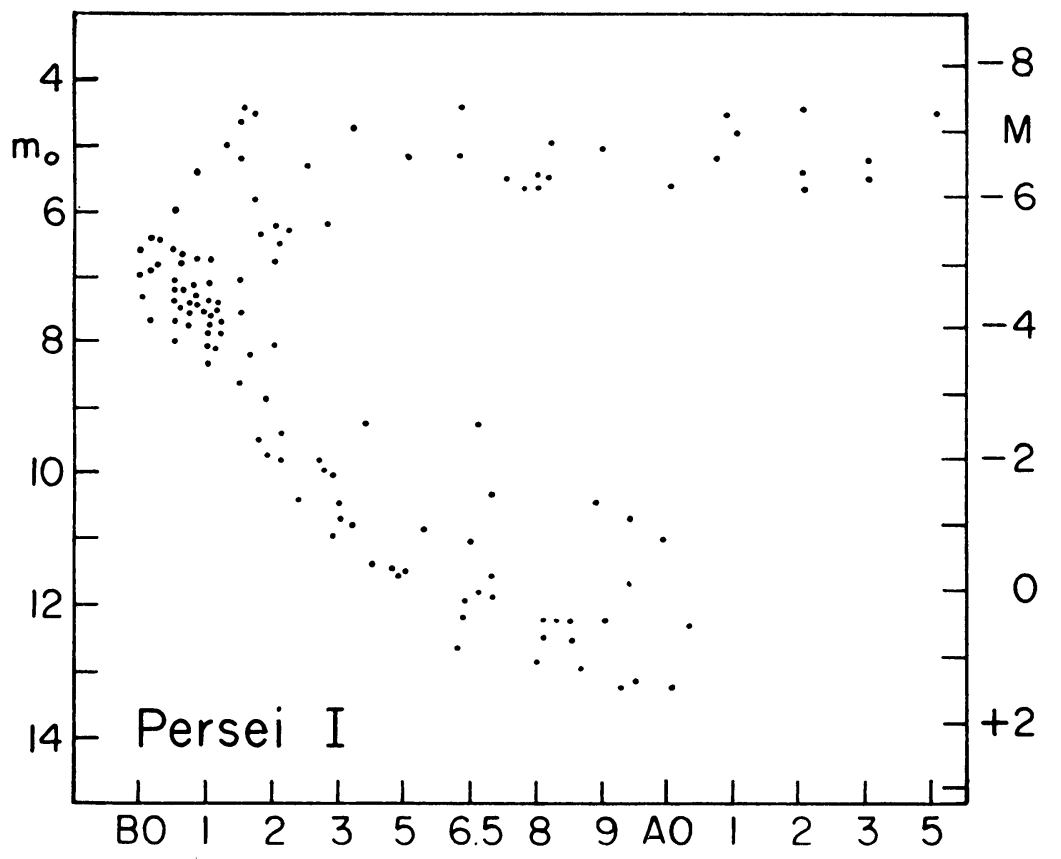

FIGURE 4. The HR diagram for the association, Perseus I.

contradistinction to the upper one, is very sharp. This was first discovered by Sharpless (I954) for the association Orion I and is observed to a greater or lesser degree in practically all groups of hot stars (see Figs. 3-5).

Some of the differences between HR diagrams in Figs. I and 6 are due to the circumstance that a considerable part of stars in Fig. I (especially those of low luminosity) belong to the galactic field, whereas Fig. 6 is plotted exclusively for stars of spatial groups.

From Fig. 6 we can determine the "line of zero age", i.e. the initial main sequence for young stars which have just completed the process of gravitational contraction. The term "line of zero age" was introduced by Hiltner and Johnson (I956).

It is important to mention that in this case the position of the "line of zero age" is determined on the basis of observational data, independently of any definite hypotheses regarding the direction and rates of stellar evolution. Only the most general contemporary conceptions on the character of this evolution are taken into consideration here.

In Fig. 6 the initial main sequence should, evidently, be a line passing along the lower boundary of the composite main sequence. Indeed, stars still undergoing contraction, and stars whose evolution is proceeding by way of nuclear transformations without corpuscular radiation and without mixing, should lie to the right of this line. The "line of zero age" is represented in Fig. 6 and in Fig. I by a solid line. The "line of zero age" obtained here lies considerably lower than the line determined by Hiltner and Johnson (I956) and by Johnson (I957).

An extremely important question pertaining to the problem of the origin and evolution 


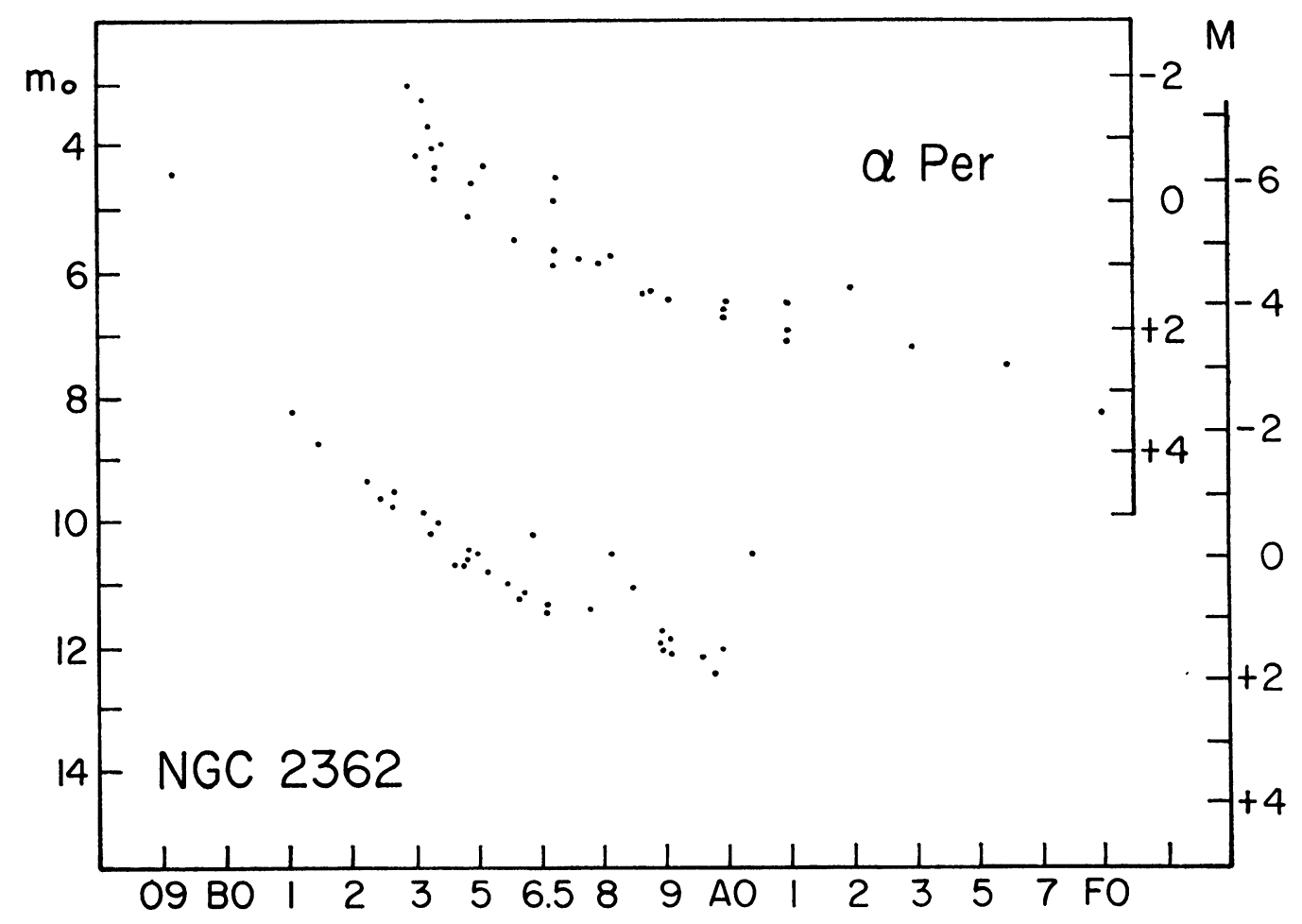

FIGURE 5. HR diagrams for the $\alpha$ Persei association and for NGC 2362.

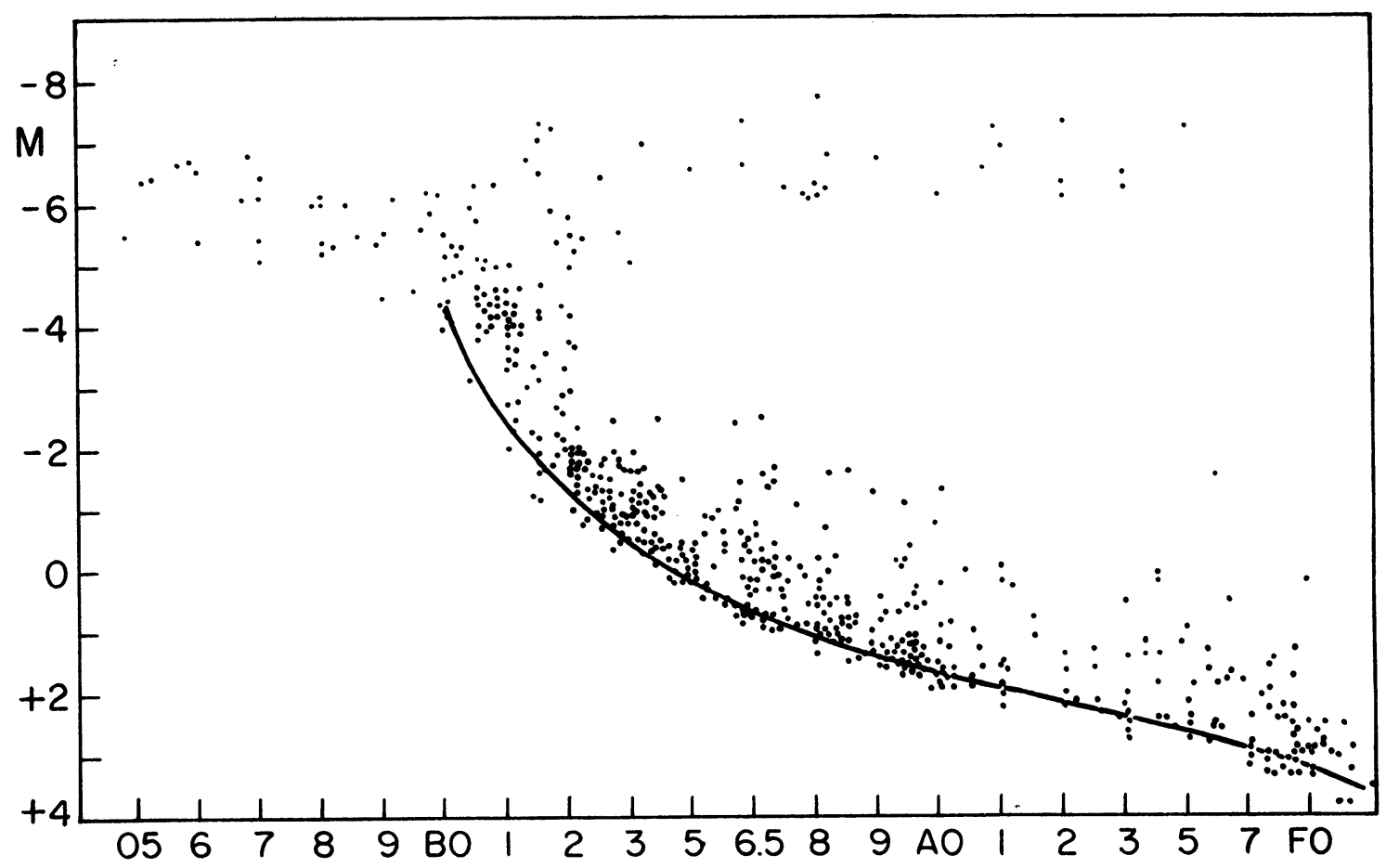

FIGURE 6. A combined HR diagram for $\mathrm{r} 3$ clusters and associations, together with the zero-age line. 
of stars is that of the population density and structure of the right half of the HR diagram for groups of hot stars and related to this, in particular, the question of the relation between the $\mathrm{O}$ - and $\mathrm{T}$ - associations.

The data of Parenago, Walker and Johnson on the structure of the HR diagram for the groups of hot stars Orion I, NGC 6530 and NGC 2264 show that in these cases the densely populated sequences of stars of the later spectral classes, starting at some one class (usually late $\mathrm{B}$ or A) deviate considerably to the right of the initial main sequence-the "line of zero age". The value of this deviation grows progressively in the direction of the later spectral classes and can be as large as $3^{\mathrm{m}}-4^{\mathrm{m}}$ and more. According to contemporary ideas on stellar evolution, these stars are still in the stage of gravitational contraction.

Fig. 7 represents the complete HR diagram for the cluster NGC 2264 (S Mon). The dots are stars of constant brightness, the circles-variables of the RW Aur type according to the data of Walker. The vertical bars give the limits of variation of the brightness (photographic).

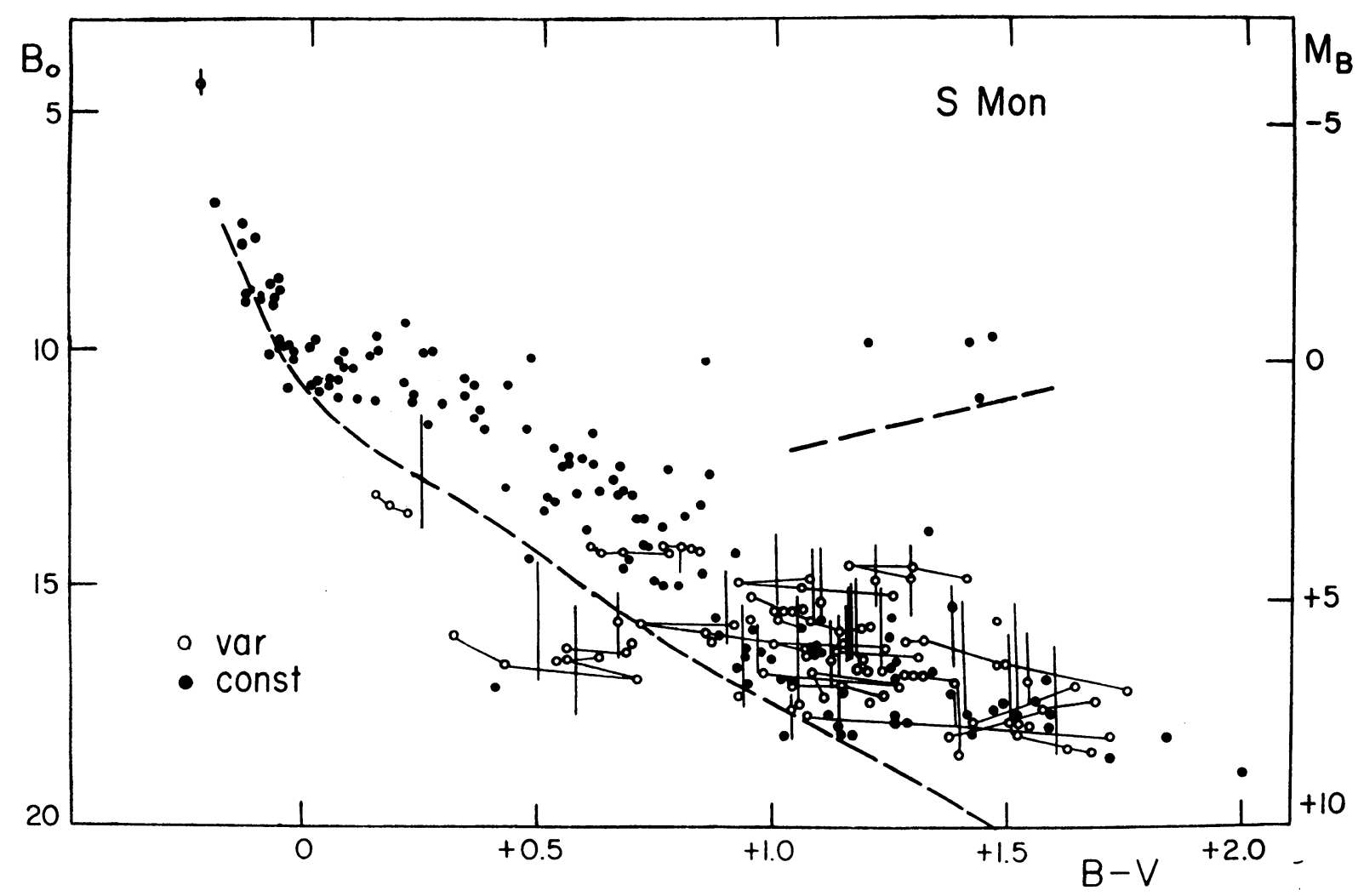

FIGURE 7. The HR diagram for the cluster NGC 2264, S Mon.

The dotted lines-here and in Figs. 8 to ro-are the initial main sequence (the "line of zero age") and the giant branch. The magnitudes (B) and the colors (B-V) of stars have not been corrected for absorption; $\mathrm{m}-\mathrm{M}=\mathrm{IO}^{\mathrm{m}}$.o.

Fig. 8 shows the HR diagram for stars in the Orion nebula (the group T Ori). The dots are stars of constant brightness (according to the data of Sharpless and Johnson). The vertical bars give the limits of variation of the brightness of variables of the RW Aur type. 
The position of variables on the abscissa axis corresponds to the mean color indices determined by Parenago. The absorption of light has been taken into account; for stars of constant brightness the correction is exact, for variables it was statistically obtained from Sharpless' curve of the dependence of $\mathrm{CE}$ on the distance from $\Theta^{1}$ Ori $\mathrm{A}$. We have put absorption $=4 \mathrm{CE}$.

Fig. 9 gives the combined HR diagram for four T-associations in Taurus - Auriga. The dots denote the stars of the Cassiopea - Taurus stream according to Blaauw. The position

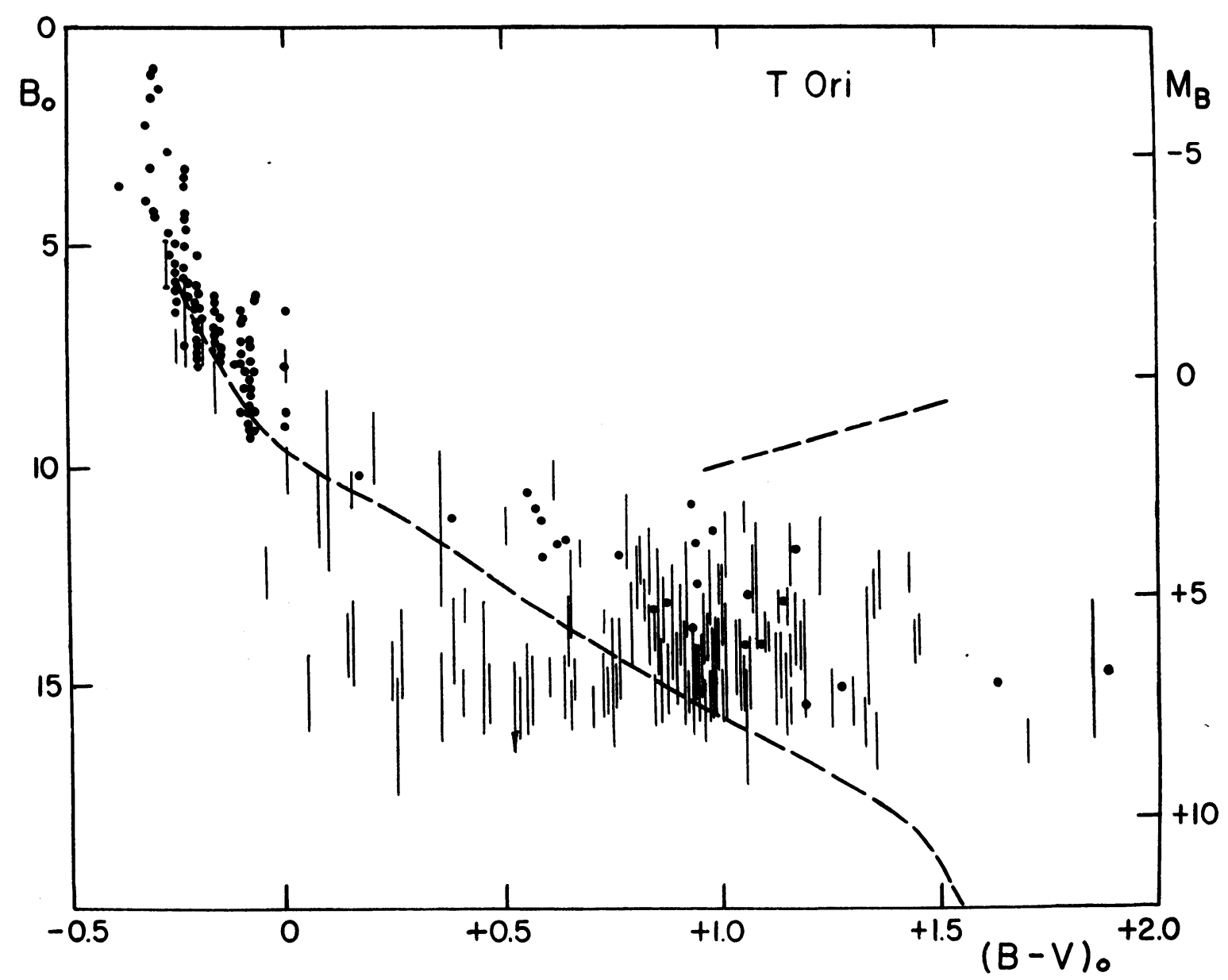

FIGURE 8. The HR diagram for the Orion nebula region, the group T Ori. The variables and their ranges are indicated by vertical bars.

of the main sequence for this stream corresponds to $r_{0}=I 50$ parsecs. The positions of the variable stars have not been corrected for absorption, which may make a difference of as much as $2^{\mathrm{m}}-3^{\mathrm{m}}$. If the absorption is taken into account the picture will correspond exactly to Figs. 7 and 8 .

We also considered all the other $\mathrm{T}$ - associations, for which such diagrams may be plotted and distances determined; these are the groups $\mathrm{CO}$ Ori, $\rho \mathrm{Oph}, \mathrm{R} \mathrm{CrA}$, and $\zeta \mathrm{Per}$ (IC 348).

On the basis of an analysis of all the known observational data relating to the HR diagrams for $\mathrm{O}$ - and $\mathrm{T}$ - associations the following conclusions may be drawn. 


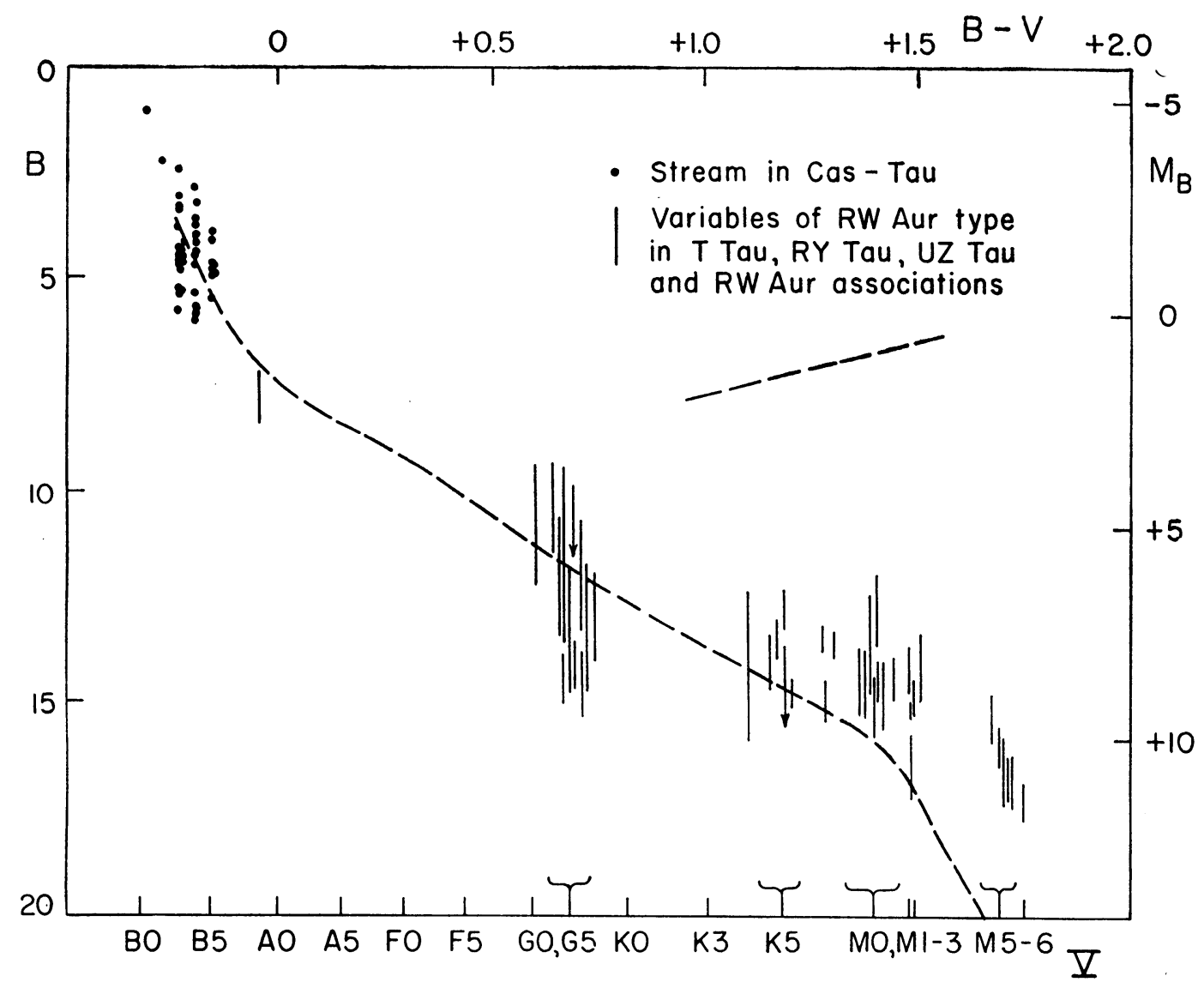

FIGURE 9. A combined HR diagram of four $\mathrm{T}$ associations.

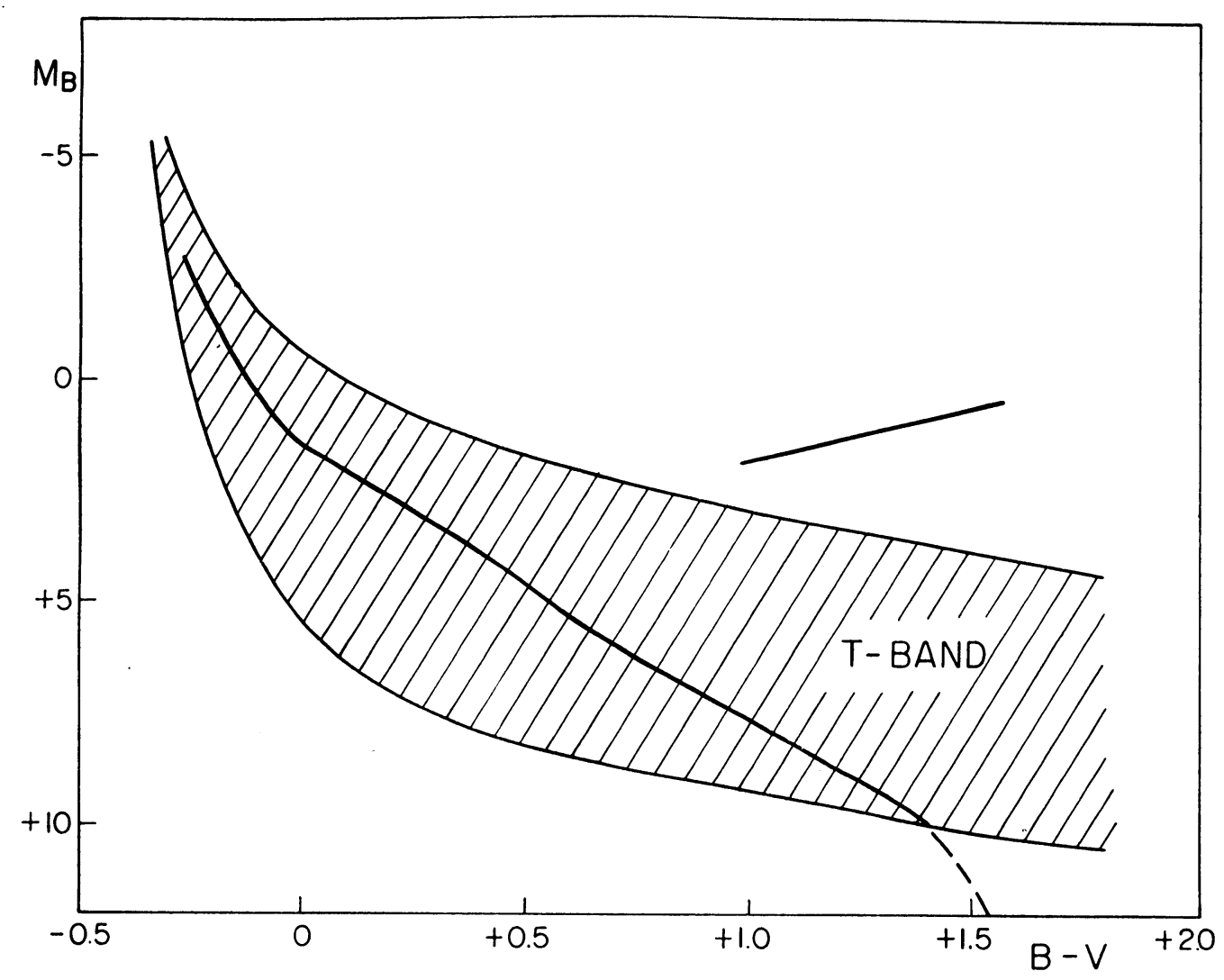

FIGURE IO. The dashed region shows the location of the variable stars of the RW Aur type. 
I. The HR diagrams for $\mathrm{T}$ - associations not explicitly related to O-associations, are identical with the diagrams for $\mathrm{T}$-associations forming a part of $\mathrm{O}$-associations.

2. Variable stars of the RW Aur type are situated on the HR diagram in the region of a wide band, which can be called the T-band (Fig. Io). They are mostly subgiants, although different from the ordinary subgiants.

3. Among the stars of the RW Aur type, objects of the most different spectral classes - from $\mathrm{O}$ to $\mathrm{M} 6$ are to be met. Variable stars of the $\mathrm{O}-\mathrm{G}$ classes can, evidently lie both above and below the main sequence.

4. With a change in brightness, variable stars of the RW Aur type move along the HR diagram on the average parallel to the general direction of the T-band.

The observational data discussed above by no means exhaust all the properties and peculiarities of the HR diagram for such spatial groups as $\mathrm{O}$ - and $\mathrm{T}$-associations.

We have drawn attention only to those of the principle features of the HR diagrams for these groups which, in our opinion, may have some bearing on the problem of the origin and evolution of stars in general and in particular, the stars of the flat component of the Galaxy.

The present communication is based on the results of the following two papers :

[I] I. M. Kopylov, Publ. Crimean Astrophys. Obs., 20, 1958.

[2] P. N. Kholopov, Astron. J. USSR, 35, No. 3, 1958. 